\title{
FoxH1 (Fast) functions to specify the anterior primitive streak in the mouse
}

\author{
Pamela A. Hoodless, ${ }^{1,4}$ Melanie Pye, ${ }^{1}$ Claire Chazaud, ${ }^{1}$ Etienne Labbé, ${ }^{2}$ Liliana Attisano, ${ }^{2}$ \\ Janet Rossant, ${ }^{1,3}$ and Jeffrey L. Wrana ${ }^{1,3,5}$ \\ ${ }^{1}$ Samuel Lunenfeld Research Institute, Mount Sinai Hospital, Toronto, Ontario, Canada M5G 1X5; ${ }^{2}$ Department of Anatomy \\ and Cell Biology and ${ }^{3}$ Department of Medical Genetics, Medical Sciences Building, University of Toronto, Toronto, \\ Ontario, Canada M5S 1A8
}

The node and the anterior visceral endoderm (AVE) are important organizing centers that pattern the mouse embryo by establishing the anterior-posterior (A-P), dorsal-ventral (D-V), and left-right (L-R) axes. Activin/nodal signaling through the Smad2 pathway has been implicated in AVE formation and in morphogenesis of the primitive streak, the anterior end of which gives rise to the node. The forkhead DNA-binding protein, FoxH1 (or Fast), functions as a Smad DNA-binding partner to regulate transcription in response to activin signaling. Here, we show that deletion of FoxH1 in mice results in failure to pattern the anterior primitive streak (APS) and form node, prechordal mesoderm, notochord, and definitive endoderm. In contrast, formation of the AVE can occur in the absence of FoxH1. The FoxH1 mutant phenotype is remarkably similar to that of mice deficient in the forkhead protein Foxa2 (HNF3 $\beta$ ), and we show that Foxa2 expression is dependent on FoxH1 function. These results show that FoxH1 functions in an activin/nodalSmad signaling pathway that acts upstream of Foxa2 and is required specifically for patterning the APS and node in the mouse.

[Key Words: gastrulation; anterior primitive streak; nodal; Smad; FoxH1]

Received January 31, 2001; revised version accepted March 2, 2001.

The concept of an embryonic organizer was first introduced in the 1920s from studies on amphibian development. These studies identified a region on the dorsal side of the amphibian embryo, known as Spemann's organizer, which when transplanted to an ectopic site on the ventral side of a host embryo was able to induce a second body axis (Harland and Gerhart 1997; for review, see Davidson and Tam 2000). This organizer was able to recruit host tissues to form a complete axis, including a head, with appropriate $\mathrm{D}-\mathrm{V}, \mathrm{A}-\mathrm{P}$, and $\mathrm{L}-\mathrm{R}$ patterning.

In the mouse, the organizing center or node is a distinct group of cells located at the anterior end of the primitive streak (Davidson and Tam 2000). The node establishes the L-R axis, and cells emanating from the node form the axial mesodermal structures, the prechordal plate mesoderm and notochord, that signal D-V patterning in the embryo. The anterior primitive streak (APS) is also the source of the embryonic (or definitive) endoderm cells, which intercalate with and displace the

\footnotetext{
${ }^{4}$ Present address: Terry Fox Laboratory, B.C. Cancer Agency, 601 West 10th Avenue, Vancouver, BC, Canada V5Z $1 \mathrm{~L} 3$.

${ }^{5}$ Corresponding author.

E-MAIL wrana@mshri.on.ca; FAX (416) 586-8869.

Article and publication are at http://www.genesdev.org/cgi/doi/10.1101/ $\operatorname{gad} .881501$.
}

visceral endoderm to form the gut (Lawson et al. 1991; Wells and Melton 1999). In common with its amphibian counterpart, transplantation of the mouse node to an ectopic site can induce a secondary body axis. This secondary axis, however, lacks head structures, which suggests that the organizer is separated into two distinct entities, the node or trunk organizer and the head organizer. In the mouse, patterning of the anterior neural plate occurs prior to gastrulation and involves the visceral endoderm on the anterior side of the embryo (anterior visceral endoderm or AVE). Signals emanating from the AVE are thought to convey anterior patterning to the overlying ectoderm and induce expression of forebrain markers (Beddington and Robertson 1999). The signals involved in establishing the node and the AVE and how these organizers function to pattern the embryo are not fully understood.

Activins and the activin-like ligands, such as nodal and $\mathrm{Vg} 1$, are members of the transforming growth factor- $\beta$ (TGF $\beta$ ) family of ligands. An important role for activins in early embryonic patterning was suggested by studies in which injection of mRNA encoding activin or Vgl on the ventral side of a Xenopus embryo was sufficient to induce a second body axis (Harland and Gerhart 1997). This demonstrated that ectopic expression of ac- 
tivin family members could imitate organizer functions. Questions have remained, however, regarding how these factors function in vivo to regulate organizer activity.

Activins signal through a transmembrane serine/ threonine kinase receptor complex composed of a type II receptor, ActRIIA or ActRIIB, and a type I receptor, ActRIB (also known as ALK4) (for review, see Klüppel et al. 2000). In the presence of ligand, the type II receptor phosphorylates ActRIB, activating the type I receptor kinase, which can then transmit the signal to intracellular components. The central components of the intracellular signaling pathway for TGF $\beta$ ligands belong to a family of proteins known as Smads (Klüppel et al. 2000). In the case of activins, two receptor-regulated Smads (RSmads), Smad2 and Smad3, are capable of transducing signals. These R-Smads interact directly with and are phosphorylated by ActRIB (Hoodless et al. 1999), which induces them to form heteromeric complexes with the common Smad, Smad4. The R-Smad/Smad4 complex then translocates to the nucleus and functions to regulate transcription. Although Smads can bind directly to DNA, evidence favors a model in which Smads cooperate with DNA-binding proteins to form high-affinity, specific interactions with cognate DNA (for review, see Attisano and Wrana 2000).

FoxH1 (also known as FAST) is a forkhead or wingedhelix DNA-binding protein that was initially identified by its ability to bind to an activin response element in the promoter region of the Xenopus Mix.2 gene (Chen et al. 1996). One FoxH1 homolog has been identified in mouse (also known as FAST2 or FoxH1a), as well as in human (FoxH1) and zebrafish (schmalspur) (Labbé et al. 1998; Zhou et al. 1998; Pogoda et al. 2000; Sirotkin et al. 2000). FoxH1 forms complexes with heteromers of Smad4 and either phosphorylated Smad2 or Smad3 (Labbé et al. 1998; Hoodless et al. 1999). Binding of this complex to DNA is stabilized by Smad4 contact with DNA at Smad-binding sites that lie adjacent to the FoxH1-binding site. Interestingly, FoxH1 does not contain a transcriptional activation domain and requires Smad interaction for transcriptional regulation, probably through the recruitment of transcriptional cofactors (Attisano and Wrana 2000). In the mouse, FoxH1 is expressed throughout the epiblast prior to and during gastrulation (E6.0-E7.5), with low levels detected in the extraembryonic endoderm (Weisberg et al. 1998). At early somite stages, FoxH1 is expressed bilaterally in the lateral plate mesoderm, and expression is subsequently restricted to the heart (Weisberg et al. 1998; Saijoh et al. 2000). These expression patterns suggest that FoxH1 functions during early embryonic patterning, and in zebrafish loss of FoxH1 causes variable defects in axial structures, indicative of potential functions in modulating nodal signaling in the organizer (Pogoda et al. 2000; Sirotkin et al. 2000). Consistent with this, FoxH1 regulates the goosecoid (Gsc), lefty, nodal, and pitx2 genes, which are all expressed around the time of gastrulation (Labbé et al. 1998; Osada et al. 2000; Saijoh et al. 2000; Shiratori et al. 2001).

Disruption of activin-like signaling pathways in mice has demonstrated that this pathway has numerous functions that are critical during gastrulation (for review, see Schier and Shen 1999). Mesoderm and primitive streak formation is impaired in nodal mutant mice and in mice deficient in the type I activin receptor, $A c t R I B$, or both of the activin type II receptors, $A c t R I I A$ and $A c t R I I B$. More detailed analysis has demonstrated that $A c t R I B^{-/-}$ES cells are capable of forming mesoderm; however, primitive streak formation is impaired (Gu et al. 1998). Mutation of Smad2 also disrupts normal gastrulation, which suggests a role for an activin-like signaling pathway in early pattern formation (Nomura and Li 1998; Waldrip et al. 1998; Weinstein et al. 1998; Heyer et al. 1999). Interestingly, for two of the mutant alleles, Smad2 ${ }^{\text {Robm } 1}$ and $S m a d 2^{d e x}$, the entire epiblast differentiates into extraembryonic mesoderm, and AVE markers are absent, which suggests that Smad2 is required in the visceral endoderm to establish an A-P axis in the epiblast (Waldrip et al. 1998; Heyer et al. 1999). Chimeric analysis further demonstrated that $A c t R I B$ and nodal function in the visceral endoderm to establish A-P patterning and anterior development (Varlet et al. 1997; Gu et al. 1998). Taken together, studies in mice indicate a role for an activin/nodal signaling pathway in two aspects of early embryonic patterning, the formation of the A-P axis by establishing the AVE and the development of the primitive streak.

To understand how this pathway regulates patterning, we undertook an analysis of the role of the Smad DNAbinding partner FoxH1, by deleting the gene in mice. Homozygous FoxH1 null embryos lack a node, notochord, and prechordal plate mesoderm, resulting in fused somites and a flattened neural plate. However, anterior neural plate structures are present in mutants, but are underdeveloped. FoxH1 is not required to establish the AVE, although some mutants display a constriction in the visceral endoderm that is centered at the extraembryonic/embryonic boundary, suggesting that FoxH1 might function to regulate expansion of the visceral endoderm around the time of gastrulation. Interestingly, FoxH1 mutant embryos are remarkably similar to those deficient for the forkhead protein Foxa2, which embryos also lack midline structures, and we demonstrate that FoxH1 is required for Foxa2 expression. Finally, we show that FoxH1 is essential for development of the embryonic endoderm. Together, these studies reveal a FoxH1Foxa2 regulatory pathway that is essential for specification of the APS and regulation of axial patterning and endoderm development in the mouse.

\section{Results}

\section{FoxH1 mutants lack midline structures}

FoxH1 was initially identified in Xenopus as a transcriptional partner for Smad proteins that mediates activin/ nodal/Vg1 signals during early development, and one FoxH1 homolog has been identified in mouse. To understand what pathways require FoxH1 function during vertebrate development we undertook a genetic analysis 
and deleted the FoxH1 (Fast2) gene in the mouse. The mouse FoxH1 gene is composed of 3 exons that span $\sim 2.2$ $\mathrm{kb}$ of genomic sequence including $5^{\prime}$ and $3^{\prime}$ untranslated sequences (Fig. 1A). Because of the small size of the gene, we designed a strategy to delete the entire FoxH1 genomic sequence in ES cells to generate a complete null mutation (Fig. 1). Of note, the 3' UTR of the FoxH1 gene overlaps with the kinesin gene, KIFC2, which is encoded on the opposite DNA strand (Liu et al. 1999), and our strategy interferes with the KIFC2 gene. However, KIFC2 is neuron-specific, is not expressed in the developing mouse until embryonic day 16, and deletion of the KIFC2 gene has no overt phenotype (Yang et al. 2001). Gastrulation defects in the FoxH1 mutant embryos are therefore not caused by interference with KIFC2. Foxh1 heterozygotes were generated from two independent R1 ES cell lines and crossed with the outbred strain, CD-1. Both lines exhibited identical defects as described below.

Analysis of offspring from intercrosses of $\mathrm{FOXH1}^{+/-}$ animals revealed that no FoxH1 ${ }^{-/-}$pups were born (Fig. 1B,C; Table 1). Detailed analysis of genotypes during early embryogenesis revealed that normal Mendelian ratios were recovered up to E8.5. However, at later devel-
Table 1. Summary of genotypes of embryos from FoxH1 heterozygote matings

\begin{tabular}{lrrcr}
\hline Age & Wild type & Heterozygote & Mutant & Resorbing \\
\hline E6.5 & $79(25.8 \%)$ & $155(50.7 \%)$ & $56(18.3 \%)$ & $16(5.2 \%)$ \\
E7.5 & $57(24.5 \%)$ & $100(42.9 \%)$ & $73(31.3 \%)$ & $3(1.2 \%)$ \\
E8.5 & $47(23.0 \%)$ & $96(47.0 \%)$ & $52(25.5 \%)$ & $9(4.4 \%)$ \\
E9.5 & $5(17.2 \%)$ & $12(41.4 \%)$ & $10(34.5 \%)$ & $2(6.9 \%)$ \\
E10.5 & $6(46.2 \%)$ & $4(30.8 \%)$ & $1(7.7 \%)$ & $2(15.4 \%)$ \\
\hline
\end{tabular}

Embryos were dissected at the indicated stages from FoxH1 +/$x+/$ - matings and genotyped by PCR (see Fig. 1). Resorptions that we were unable to genotype are also indicated. The pooled data from several litters is shown. For E9.5 and E10.5, the results are from two litters and a single litter, respectively.

opmental stages FoxH1-/- embryos were undergoing resorption, and by E10.5 only one FoxH1-/- embryo was recovered. To assess the defect in FoxH1 ${ }^{-/-}$embryos we first examined the gross morphology. At E6.5, FoxH1 mutant embryos were overtly normal, although they often were slightly delayed compared to wild-type littermates. By E7.5 and early head fold stages, formation of the primitive streak, head folds, allantois, chorion, and

A

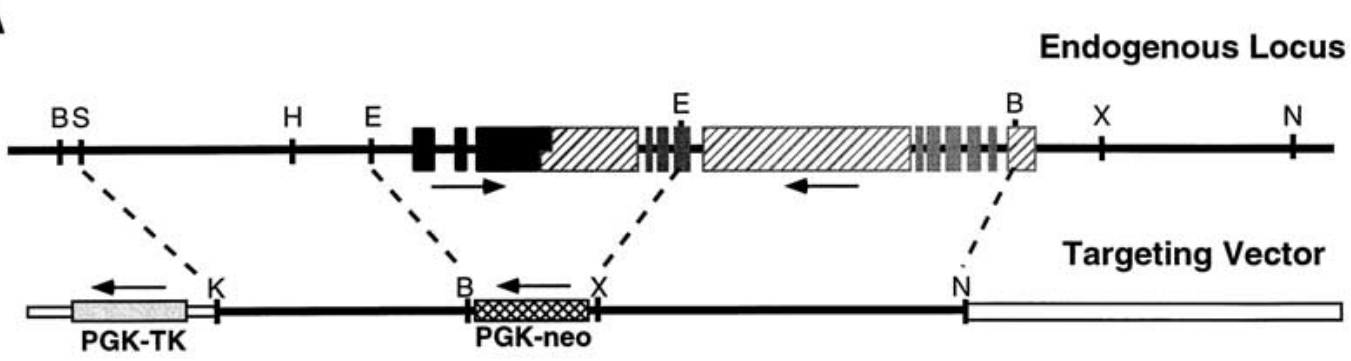

Targeted Locus

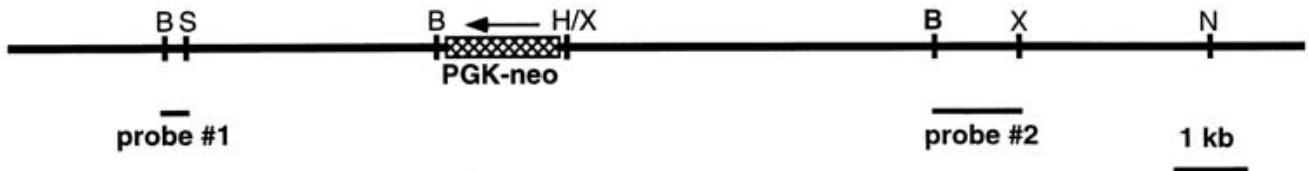

B

C
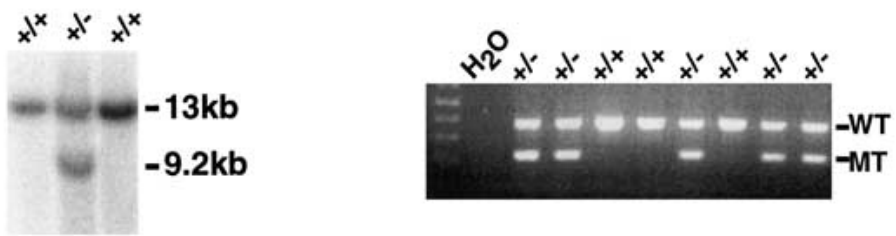

Figure 1. Strategy for deletion of the FoxH1 gene. (A) A schematic representation of the wild-type and mutant alleles and the targeting vector. E, EcoRI; H, HindIII; B, BamHI; X, XbaI; N, NotI; and S, SalI. The FoxH1 gene is composed of three exons (shown in black), and the KIFC2 gene, encoded in the opposite orientation, is shown in gray with hatched areas indicating regions where the intron/exon boundaries are not mapped. The overlapping region between FoxH1 and KIFC2 is shown as a jagged line. (B) Southern blot analysis of ES cell colonies following selection. Genomic DNA digested with NotI/HindIII was probed with the 3' flanking probe (probe \#2). The 13-kb wild-type fragment and the 9.2-kb recombinant fragment are indicated. $(C)$ PCR genotyping of the offspring of a FoxH1 heterozygote cross. No homozygous offspring were observed. The three primers amplify a 379-bp (R4 and F5) fragment from the wild-type allele and a 219-bp (R4 and CreR) fragment from the mutant allele. 
amnion was observed (Fig. 2C,D). However, unlike wildtype littermates, all of these embryos lacked a distinct node and midline. Interestingly, we observed pinching in approximately $25 \%$ of the FoxH1 $1^{-/-}$embryos examined. This pinching, which we refer to as a type II defect, centered on the extraembryonic-embryonic junction (Fig. $2 \mathrm{C}, \mathrm{D})$, was variable in extent, and in severe cases led to arrest in development of the embryonic portion while extraembryonic tissues continued to develop (not shown). It is unclear what causes these constrictions, although similar phenotypes have been observed in $\mathrm{Lim}^{-/-}$, Otx2 $2^{-/-}$, and chordin ${ }^{-/} ;$noggin ${ }^{-/-}$embryos (Shawlot and Behringer 1995; Ang et al. 1996; Bachiller et al. 2000), and notably, Foxa2 mutant mice (Ang and Rossant 1994; Weinstein et al. 1994). In the less severely affected or nonaffected FoxH1 $1^{-/-}$embryos, development of the embryonic region proceeded, and at E8.5, somites formed and development of a heart tube was observed (Fig. 2F). Strikingly, inspections of the midline revealed that all of these FoxH1 mutant embryos (of 113 examined) had fused somites and absent midline structures (Fig. $2 \mathrm{H}, \mathrm{J})$. In addition, there were abnormalities in the anterior regions when compared to wild-type littermates
(Fig. 2F). These mutant embryos, which we refer to as type I mutants, also failed to develop neural folds and displayed a flat neural plate (Fig. 2H). The consequences of these defects were most apparent in the single E10.5 type I mutant we recovered, in which a single proboscislike structure was observed anterior to the midbrain/ hindbrain junction (Fig. 2K). Although a heart tube was present, heart looping failed to occur in this embryo, and the pericardiac cavity was enlarged.

To confirm the lack of midline structures, $\mathrm{FOXH}^{-/-}$ type I mutants were examined histologically at E7.5 and E8.5. At E7.5, the morphology of type I mutants was similar to wild-type embryos in both the proximal and distal regions of the embryo (Fig. 3A-E). However, in the anterior and trunk regions of mutants at E8.5, the neural plate was flat, the somites were fused across the midline, and there was no evidence of notochord in the proximity of the neural plate (Fig. 3F-I). All embryos had foregut and hindgut invaginations, and the morphology of the tail bud region was similar to that of the wild-type controls (cf. Fig. 3G,I with 3J,L). These results suggest that FoxH1 is required for formation of midline structures during mouse development.
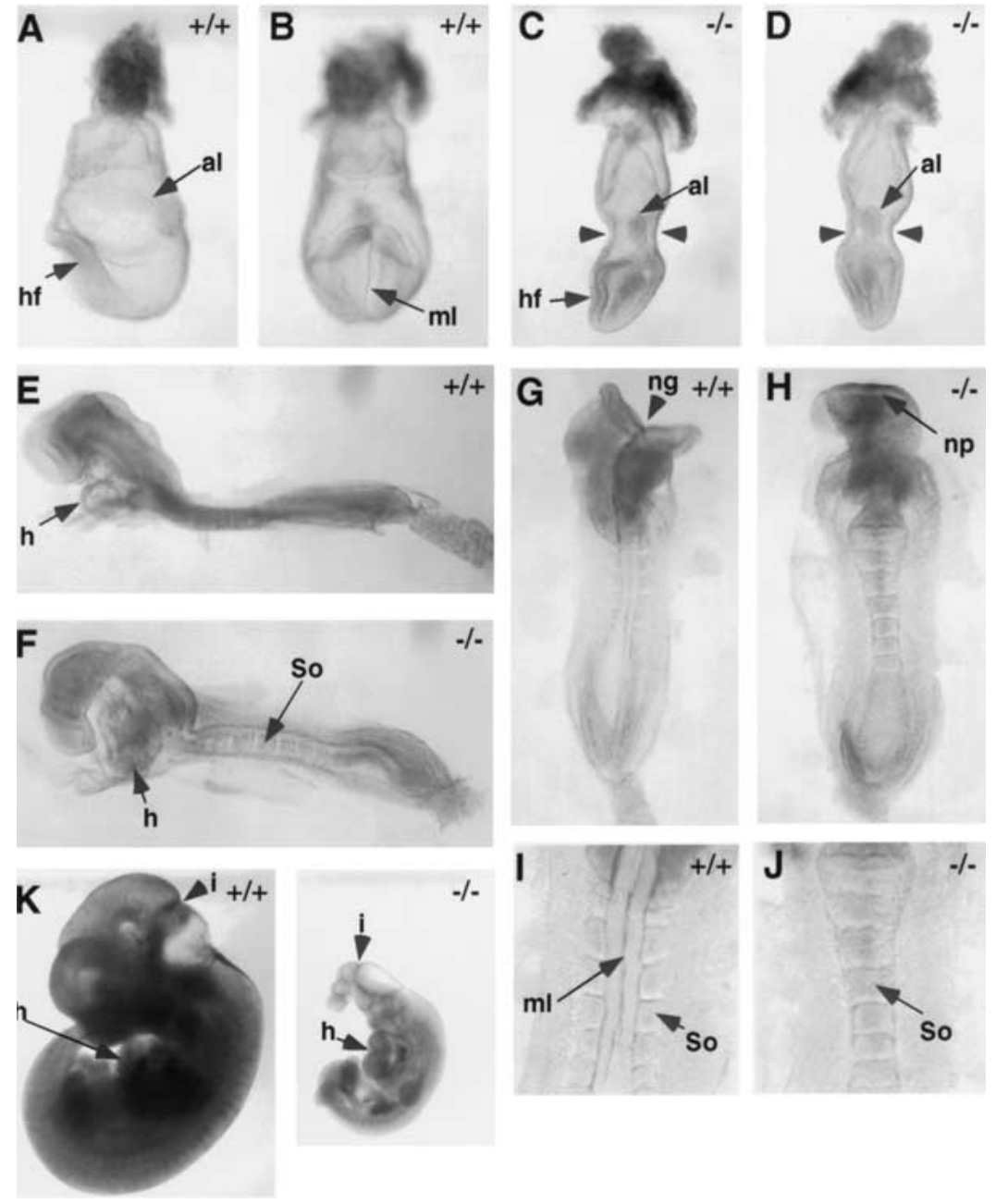

Figure 2. FoxH1 mutant embryos lack midline structures. $(A-D)$ Lateral $(A, C)$ and anterior $(B, D)$ views of wild-type $(A, B)$ and FoxH1 mutant $(C, D)$ embryos at E7.5. The FoxH1 mutants lack a midline $(\mathrm{ml})$ and a definite node. The headfolds (hf) are present but poorly developed. The allantois is also present in the mutants (al). In some embryos, pinching is observed at the junction between the embryonic and extraembryonic regions of the embryo (arrowheads in $C, D)$. $(E-J)$ Lateral $(E, F)$ and dorsal $(G-J)$ views of wild-type $(E, G, I)$ and FoxH1 mutant $(F, H, J)$ embryos at E8.5. The FoxH1 mutant embryos lack midline structures resulting in fused somites (So) and a flattened neural plate (np) compared to the wildtype embryos with a distinct neural groove (ng) and midline $(\mathrm{ml}) .(I, J)$ Show the somites at higher magnification. The FoxH1 mutant embryos also exhibit aberrant anterior head structures $(E, F)$ and have heart (h) defects because looping does not occur and the pericardial membrane is enlarged. $(K)$ Lateral view of wild-type (left) and FoxH1 mutant (right) embryos at E10.5. The mutant embryo is significantly smaller and lacks normal structures anterior to the midbrain-hindbrain junction (i), resembling mutants with holoprosencephaly. Heart looping still has not occurred in the FoxH1 mutant embryo. 

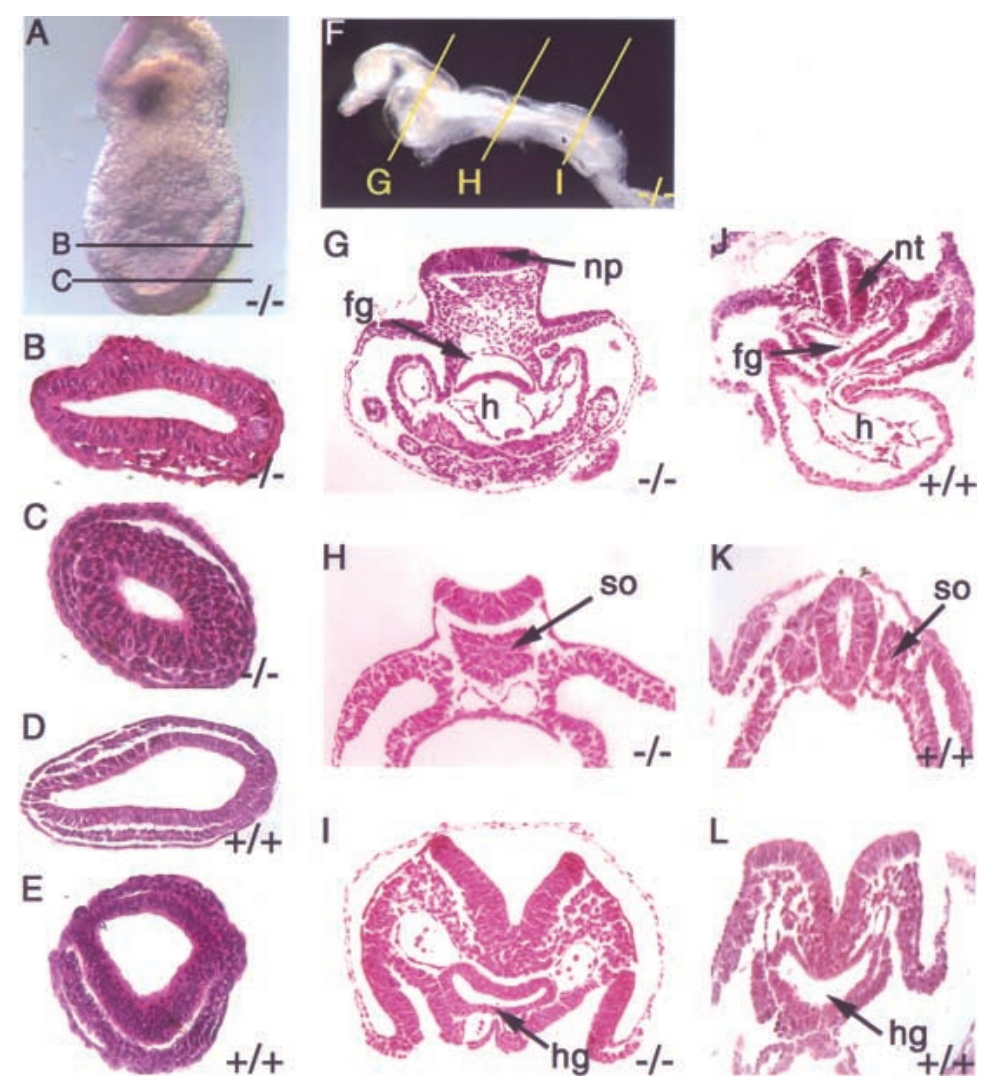

Figure 3. FoxH1 mutant embryos have fused somites and a flat neural plate. $(A)$ Lateral view of an E7.5 FoxH1 type I mutant showing the approximate location of the sections presented in $B$ and $C .(B, C)$ Proximal $(B)$ and distal $(C)$ transverse sections of the mutant embryo shown in $A$. The streak region is to the right. $(D, E)$ Transverse sections through the proximal $(D)$ and distal $(E)$ regions of a wild-type E7.5 embryo. (F) Lateral view of an E8.5 FoxH1 type I mutant embryo showing the approximate location of the sections presented in $G-I$. $(G-I)$ Sections through the anterior foregut and heart region $(G)$, midgut region $(H)$, and posterior region $(I)$ of the E8.5 FoxH1 mutant shown in $F$. $(J-L)$ Sections through the anterior foregut and heart region $(J)$, midgut region $(K)$, and posterior region $(L)$ of a wild-type E8.5 embryo. At E7.5 the type I mutants are similar to wildtype embryos, but at E8.5 all mutants lack a notochord and display flat neural plates (np), heart (h) looping defects, and fused somites (so). Foregut (fg) and hindgut (hg) invaginations are present.

\section{FoxH1 mutants fail to form axial mesendoderm}

To investigate axial patterning in the FoxH1 mutant embryos, we examined brachyury expression, which marks the nascent mesoderm in the primitive streak region and axial mesoderm during gastrulation. At E7.5 expression of brachyury was normal in the streak of type I FoxH1 mutant embryos, but there was no detectable expression in the midline region (Fig. 4B). We also examined brachyury expression in type II mutants at this stage. Although these embryos were morphologically disturbed, regionalized expression of brachyury (Fig. 4C) and nodal (data not shown) was observed on the presumptive posterior side. These results indicate that normal A-P patterning is initiated in both type I and type II mutants. Further analysis of brachyury in type I mutants revealed strong expression in the primitive streak of E8.5 mutants, but notochord staining was absent (Fig. 4F), although in some embryos we observed a brachyury-positive notochord remnant that was restricted to the posterior region ( 2 out of 10 embryos; Fig. 4E). These data suggest that notochord formation is severely impaired in type I FoxH1 mutant embryos with the majority of the embryos completely lacking a notochord.

The absence of midline structures in the FoxH1 mutant embryos was startlingly similar to the phenotype observed attributable to embryonic loss of Foxa2 (HNF3 $\beta$ ). Therefore, we also examined Foxa2 expression, which is localized to the APS, node, and developing mesendoderm of the head process at E7.5 (Ang et al.
1993; Ruiz i Altaba et al. 1993; Filosa et al. 1997). However, in both type I and type II FoxH1 mutants there was no detectable Foxa2 expression at this stage (Fig. 4H-J). At E8.5, when Foxa2 is strongly expressed in the notochord, floorplate, prechordal plate mesoderm, and developing foregut, we observed a complete absence of Foxa2 expression in the axial region of the mutants (Fig. 4L). Finally, we examined goosecoid expression, which marks the prechordal plate mesoderm at this stage. In late headfold stage embryos, goosecoid was expressed in the prechordal plate mesoderm in wild-type embryos (Fig. 4M); however, in the mutants we were unable to detect any goosecoid expression (Fig. 4N). Thus, FoxH1 functions upstream of Foxa2 and goosecoid to specify anterior mesendoderm during axial patterning in the mouse.

\section{FoxH1 mutant embryos express markers of anterior neural patterning}

Analysis of the FoxH1 ${ }^{-/-}$mice suggested that anterior development was initiated in these embryos. To examine this in more detail we analyzed expression of several markers of anterior patterning of the neural plate. Engrailed-2 (En-2) is a marker of the hindbrain-midbrain region. In all FoxH1 mutant embryos examined, En-2 was expressed across the flat neural plate (Fig. 5B,D), and the A-P boundaries of En-2 expression were similar to those of wild-type embryos (Fig. 5C,D). Next we examined Six3, which is expressed in the anterior region of the 

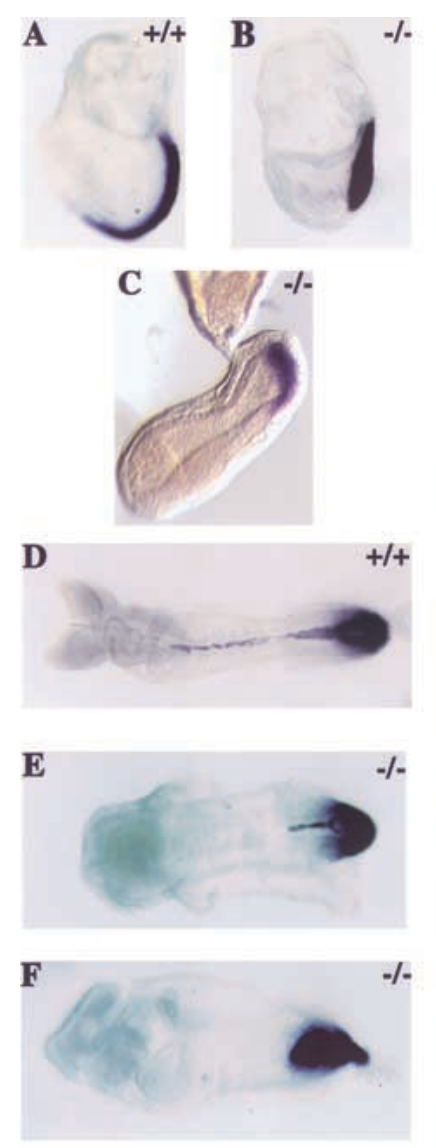
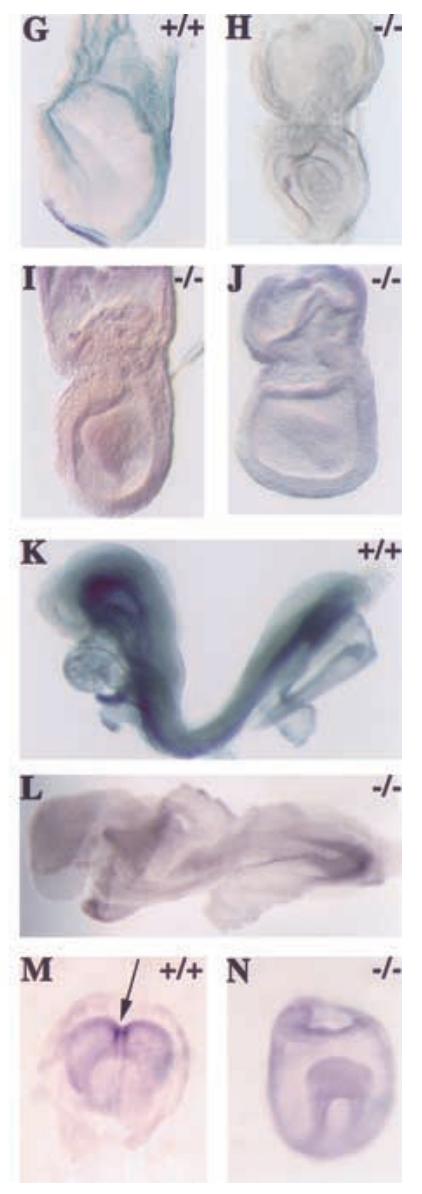

Figure 4. Expression of the axial mesoderm markers brachyury, Foxa2, and goosecoid in FoxH1 mutant embryos. $(A-C)$ Expression of brachyury in wild-type $(A)$, type I $(B)$, and type II (C) FoxH1 mutant embryos at early headfold stage. Staining is observed in the primitive streak region of FoxH1 mutant embryos but no axial mesoderm is present. $(D-F)$ Expression of brachyury in wild-type $(D)$ and type I FoxH1 mutant $(E, F)$ embryos at E8.5. Notochord expression is absent in most mutant embryos. However, some mutants display a short notochord remnant in the posterior region $(F) .(G-J)$ Expression of Foxa2 in wild-type $(G)$ and mutant $(H-J)$ embryos at early headfold stage. Foxa2 is not expressed in FoxH1 mutant embryos at this stage. $(K, L)$ Expression of Foxa2 in wild-type $(K)$ and mutant $(L)$ embryos at E8.5. Foxa2 is not expressed in midline structures. $(M, N)$ Expression of Gsc in the prechordal plate mesoderm of wild-type $(M)$ and FoxH1 mutant $(N)$ embryos at late headfold stage. Gsc expression is absent in FoxH1 mutant embryos.

neural plate marking the prospective forebrain. Although the forebrain region of the neural plate was reduced in size relative to wild-type embryos, Six3 expression was detected at low levels in the anterior region of $50 \%$ of $\mathrm{FoxH}^{-/-}$embryos (Fig. 5F), indicating that some forebrain patterning is present in the FoxH1 mutants. Similarly, Pitx2 expression, which marks Rathke's pouch (Hermesz et al. 1996), was also expressed in FoxH1 mutants (Fig. 5H). In addition to Rathke's pouch, Pitx2 is expressed in the left lateral plate mesoderm, and its expression is directly initiated by FoxH1 in response
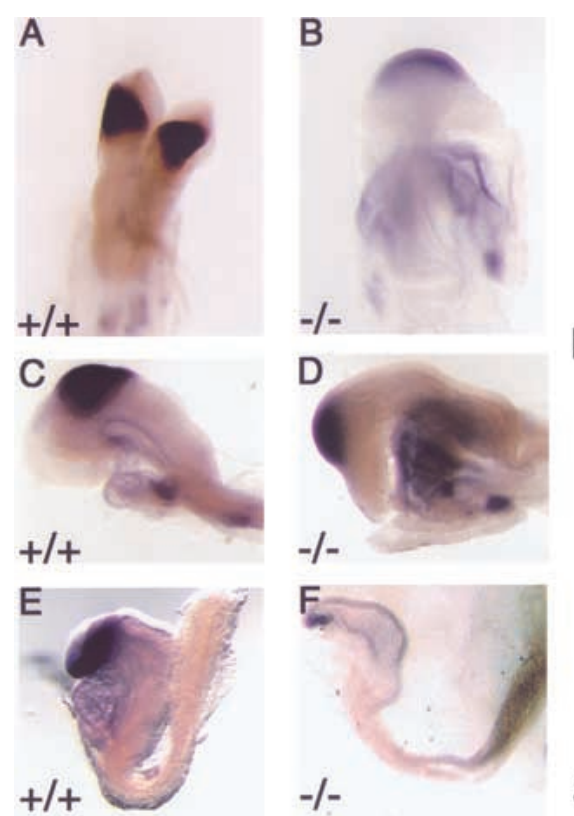

En-2
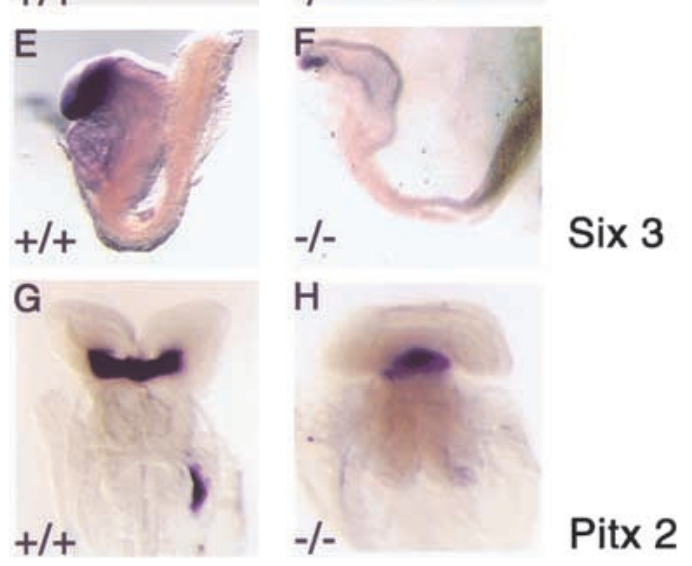

Figure 5. FoxH1 mutant embryos express markers of neural plate patterning. $(A-D) E n-2$ expression in wild-type $(A, C)$ and FoxH1 mutant $(B, D)$ embryos from a dorsal $(A, B)$ and lateral $(C, D)$ viewpoint. En-2 is expressed normally at the midbrainhindbrain junction in FoxH1 mutants. $(E, F)$ Expression of the prospective forebrain marker Six3 in wild-type $(E)$ and FoxH1 mutant $(F)$ embryos at E8.5. Weak expression of Six3 is observed in the anterior region in approximately half of the FoxH1 mutant embryos. $(G, H)$ Pitx2 expression in Rathke's pouch in wildtype $(G)$ and FoxH1 mutant $(H)$ embryos at E8.5. Normal expression of Pitx2 is observed in Rathke's pouch of FoxH1 mutant embryos. However, no expression is observed in the left lateral plate mesoderm of the heart.

to nodal signaling (Shiratori et al. 2001). Consistent with this, Pitx2 expression in the left lateral plate mesoderm was absent in FoxH1 mutant embryos (Fig. 5H). Together, these results indicate that anterior patterning of the neural plate initiates normally in the FoxH1 mutants.

\section{The AVE is patterned normally in FoxH1 mutants}

In the mouse, the AVE is initially formed at the distal tip of the visceral endoderm in response to proximal-distal signals in the embryo (for review, see Beddington and Robertson 1999|. Subsequently the AVE migrates to the anterior side of the embryo, where it specifies the A-P axis of the developing embryo and initiates anterior patterning of the neural plate. Formation of the AVE de- 
pends on signaling through the nodal-Smad2 pathway (Waldrip et al. 1998). Therefore we examined the markers Hex and cerberus-like (Cer-1), which are expressed in the AVE at E6.5 (Belo et al. 1997; Thomas et al. 1998). In FoxH1 mutants, both Hex and Cer-1 were expressed in the AVE in a similar pattern to wild-type embryos (Fig. 6A-D). Furthermore, in mutant embryos at an earlier stage of development, we observed expression of Cer-1 at the distal tip, where patterning of the AVE is initiated (data not shown). Finally, we examined Foxa2 and goosecoid, both of which are expressed in the anterior end of the primitive streak at this stage. Consistent with our previous results, we detected no expression of Foxa2 or goosecoid in the FoxH1 mutant embryos (Fig. 6F,H). These results demonstrate that FoxH1 is not critical for patterning the AVE and the A-P axis of the mouse, but is required for Foxa2 and goosecoid expression. This suggests that FoxH1 regulates specification of the APS.

\section{FoxH1 is required in the epiblast for axial patterning}

Despite the presence of AVE markers, FoxH1 mutant embryos display anterior abnormalities (Fig. 2). We wanted to determine whether FoxH1 is required in the AVE for normal anterior development or whether these anterior defects are the result of the lack of midline structures. Thus, we generated chimeric embryos in which the visceral endoderm was composed of $\mathrm{FoxH}^{-/-}$ cells, whereas the embryo was composed of both wildtype and FoxH1 ${ }^{-1-}$ cells. For this we aggregated morulae

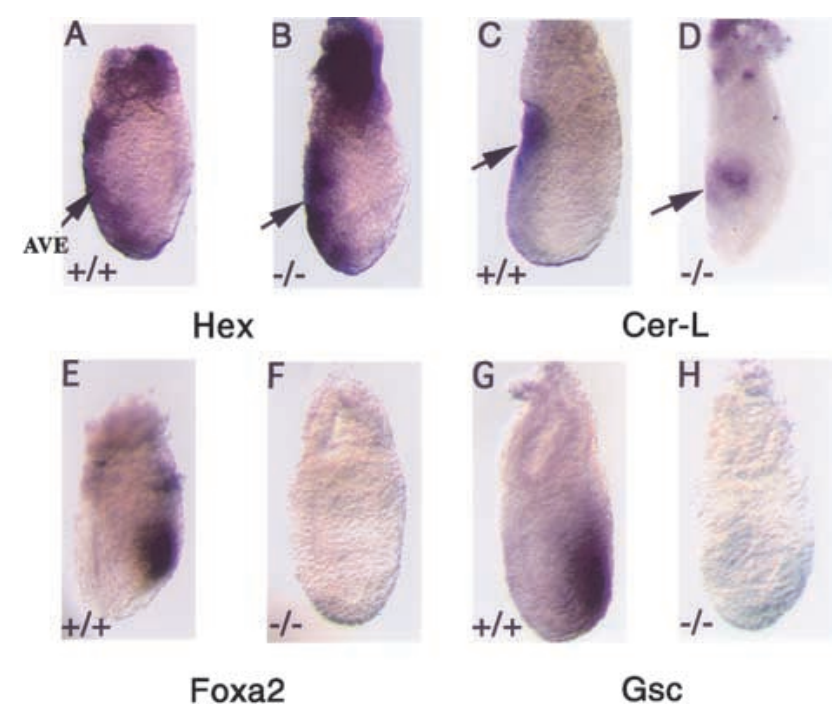

Figure 6. AVE formation occurs in FoxH1 mutant embryos, but APS markers are absent. The AVE markers Hex $(A, B)$ and Cer-1 $(C, D)$ are expressed in FoxH1 mutant embryos $(B, D)$. Wildtype embryos are shown in $A$ and $C$. In contrast, markers of the APS, Foxa2 $(E, F)$ and Gsc $(G, H)$ are absent in FoxH1 mutant embryos $(F, H)$. Wild-type littermates are shown in $E$ and $G$. generated from Foxh1 heterozygote matings with KT4 $(+\mid+)$ ES cells, which were derived from a mouse carrying the ROSA-26 lacZ gene trap and constitutively express a cytoplasmic form of $\beta$-galactosidase. This allows their contribution to various embryonic structures to be traced in the chimeric embryos (Tremblay et al. 2000). ES cells are unable to contribute to extraembryonic and primitive endoderm lineages in chimeric embryos but efficiently integrate into embryonic tissues (Beddington and Robertson 1989). Aggregated embryos were implanted in recipient mothers and dissected at E8.5-9.5. The genotype of the original morulae was determined by PCR of parietal endoderm DNA of chimeric embryos. Of 126 embryos dissected, 24 were resorbing and 20 were mutants. In aggregations of KT4 cells with wild-type embryos, we observed mixed contribution of KT4 cells to all of the embryonic structures examined, including the notochord and foregut (Fig. 7A,B,E,F,I). Of the 20 mutant embryos we recovered, 3 had no KT4 contribution. Of these, 1 was a type II mutant and 2 were of the type I phenotype. In the remaining 17 chimeric embryos, 9 displayed a type II phenotype, consistent with our observation that FOxH1 can function to regulate proliferative expansion of the visceral endoderm. Of the remaining 7 chimeric embryos, all displayed unfused somites and had a rescued notochord (data not shown). One of these (Fig. 7C) was examined by histology. This showed the presence of normal neural tube and axial structures (Fig. 7D) and, in particular, the presence of a notochord (Fig. 7G). Examination of the contribution of KT4 cells to various tissues showed that $\beta$-galactosidase-positive and -negative cells contributed well to most embryonic structures, including the neural tube (Fig. 7G,H). In contrast, when we examined the notochord, we found that it was composed exclusively of cytoplasmic stained $\beta$-galactosidase-positive cells (Fig. 7K,L). These results suggest that FoxH1 is required for axial mesoderm formation and specification of the notochord.

To examine the requirement for FoxH1 in axial mesoderm formation in greater detail, we generated wild-type and $\mathrm{FoxH}^{-/-}$ES cells from blastocysts derived from FoxH1 $^{+/}$matings (Fig. 8A). These ES cells were then aggregated with morulae generated from a ROSA-26lac $Z$ background. In these experiments, high numbers of ES cells were used in the aggregation in order to generate chimeric embryos in which the morula-derived $(+/+)$ cells were excluded. Analysis of chimeric embryos generated using wild-type ES cells revealed that the cells contributed efficiently to the embryo (Fig. 8B), and histological analysis showed that axial patterning and neural tube formation occurred normally in these embryos (Fig. 8C-E). No ES cells contributed to the visceral endoderm, confirming the lineage restriction of ES cells to embryonic tissues. Next, we introduced high levels of FoxH1 ${ }^{-/-}$ES cells into wild-type morulae, to get efficient contribution to the embryo. In contrast to wild-type ES cells, we observed a phenotype that very closely resembled the FoxH1-1- mutant mice (12 out of 12 embryos recovered). Moreover, histological evaluation showed that most regions of the embryo lacked a noto- 

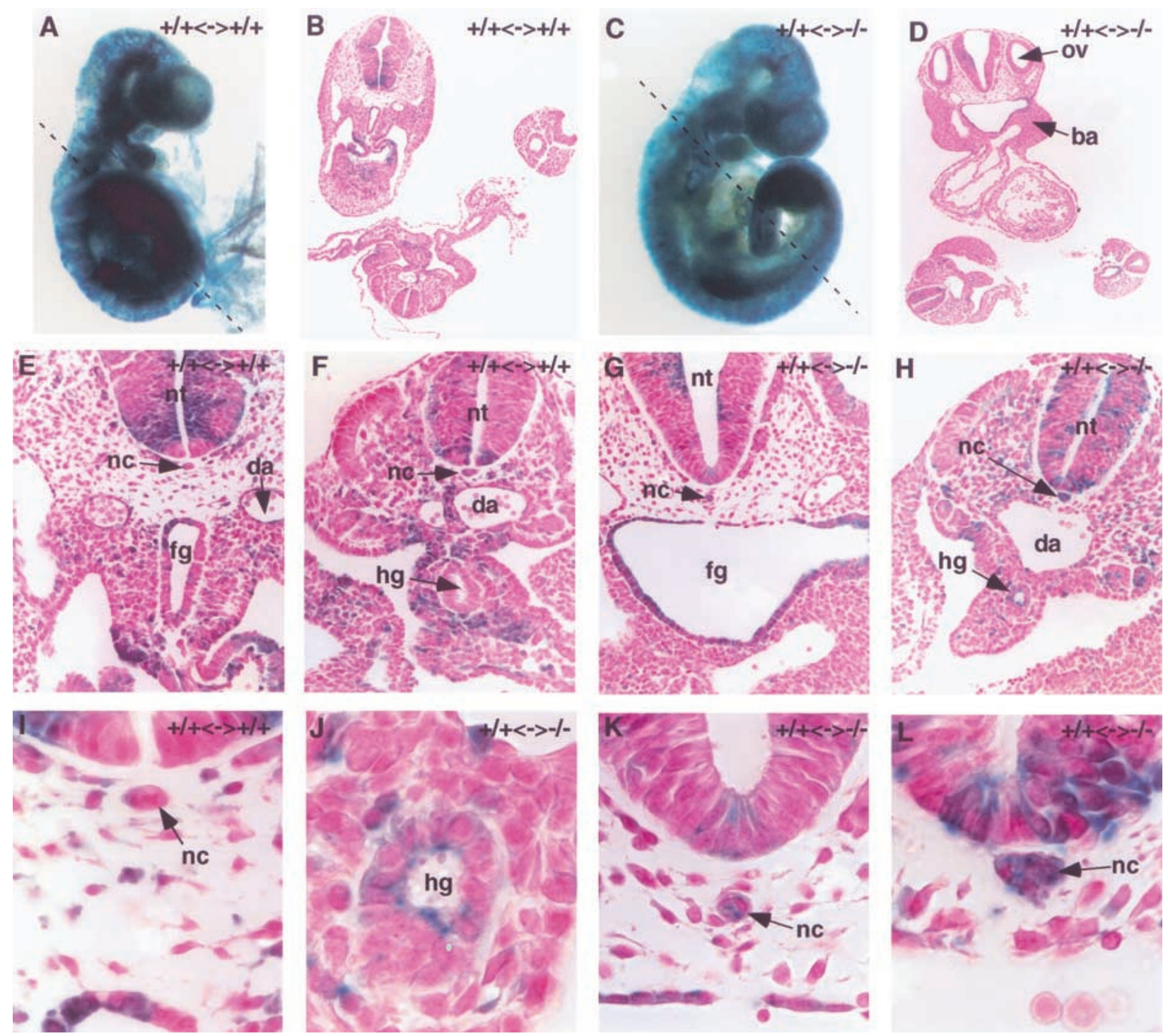

Figure 7. Wild-type ES cells can rescue anterior defects of FoxH1 mutant embryos. Wild-type KT4 ES cells containing the ROSA26lacZ gene trap were aggregated with morulae derived from a cross of heterozygous mutants for the FoxH1 mutant allele. Chimeric embryos were dissected between E8.5 and E9.5 and genotyped by PCR from the parietal endoderm. Wild-type $(A)(+/+\leftrightarrow+/+)$ and FoxH1 $(C)(+/+\leftrightarrow-/-)$ mutant morulae are shown. Normal anterior development is observed in the $+/+$ ES cell $\leftrightarrow F o x H 1^{-/-}$chimera. (B) Section through the $+/+\leftrightarrow+/+$ chimeric embryo shown in $A$. (D) Section through the $+/+\leftrightarrow-/-$ chimeric embryo shown in $C$. An otic vesicle (ov) and branchial arch (ba) are marked. (E) Enlargement of the foregut (fg) region of the section shown in $B$ showing contribution of both KT4 cells (blue) and morula cells (pink) to all tissues including the neural tube (nt), foregut, notochord (nc), and dorsal aorta (da). An enlargement of the notochord is shown in $I .(F)$ Enlargement of the hindgut (hg) region of the section shown in $B$. The hindgut is composed of a mixture of pink and blue cells. $(G)$ Enlargement of the foregut region of the section shown in $D$ showing contribution of both KT4 cells (blue) and FoxH1 mutant cells derived from the morula (pink) to most tissues. In contrast to $E$, the foregut is exclusively composed of KT4 cells. An enlargement of the notochord in $K$ shows FoxH1 mutant cells are also unable to contribute to the notochord. Note that the nuclei are stained pink because of the predominant cytoplasmic localization of $\beta$-galactosidase protein in this gene-trap line. $(H)$ Enlargement of the hindgut region of the section shown in $D$. As was observed in the foregut region. FoxH1 mutant cells are unable to contribute to the notochord (enlarged in $L$ ). The hindgut is primarily composed of wild-type KT4 cells (enlarged in J); however, a few mutant cells are observed in the hindgut.

chord, had a fused midline, and failed to form a neural fold (Fig. 8G-I). Occasionally we did observe small anterior regions in these chimeric embryos that displayed notochordlike structures immediately adjacent to the foregut and the formation of neural folds (Fig. 8J). This may be owing to signals arising from the wild-type foregut, which can pattern the overlying mesoderm (Vesque et al. 2000). Together, these results demonstrate that FoxH1 is required in the epiblast for development of normal axial mesoderm and notochord. 


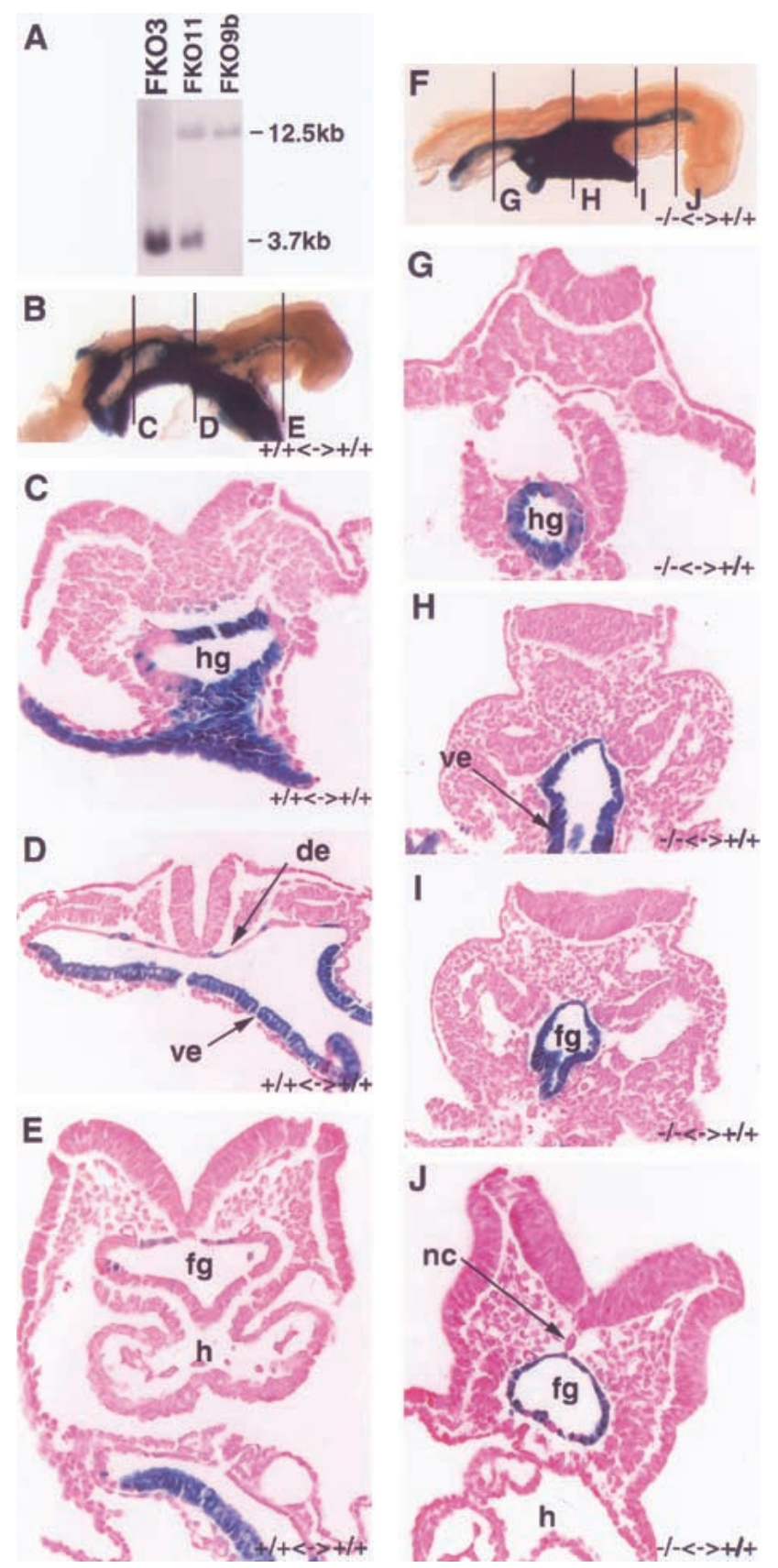

FoxH1 is required for formation of the definitive endoderm

During development, visceral endoderm cells are displaced by definitive or embryonic endoderm that arises from the anterior region of the primitive streak and forms the gut of the embryo (Lawson et al. 1991; Wells and Melton 1999). In our chimeric analyses, we observed that when wild-type KT4 ES cells were introduced into Fox $H 1^{-/-}$embryos they provided a virtually exclusive contribution to the gut endoderm (Fig. 7G,K). Contribution to the foregut and midgut was nearly absolute, whereas in the hindgut, a few Fox $\mathrm{H}^{-/-}$cells were present (Fig. 7J). The strong contribution of wild-type ES
Figure 8. FoxH1 mutant ES cells are unable to contribute to embryonic endoderm. Southern blot to genotype three ES cell lines, FKO3 (-/-), FKO11 (+/-), and FKO9b (+/+). DNA was digested with BamH1 and probed with a 200-bp BamH1/Sal1 fragment (probe \#1 in Fig. 1). The wild-type band is $12.5 \mathrm{~kb}$, and the FoxH1 mutant band is $3.7 \mathrm{~kb}$. $(B-J)$ Wild-type ROSA26 morulae that contain a ubiquitously expressed $L a c Z$ gene, were aggregated with either wild-type; FKO9b $(B-E)(+/+\leftrightarrow+/+)$; or FoxH1 $1^{-/}$, FKO3 $(F-J)$ $(-/-\leftrightarrow+/+)$, ES cells. Embryos were dissected at E8.5, and the ROSA26-derived cells were stained for $\beta$-galactosidase. Sections are shown through the hindgut $(\mathrm{hg})$ region $(C, G)$, midgut region $(D, H)$, and the foregut $(\mathrm{fg})$ region $(E, I, J)$. Aggregations with $\mathrm{FKO} 9 \mathrm{~b}$ $(+/+\leftrightarrow+/+$, pink cells) show extensive contribution to the embryo in all tissues. A few wild-type (blue) cells contribute to the foregut, midgut, and hindgut. Aggregations with FKO3 $(-/-\leftrightarrow+/+)$ demonstrate that $\mathrm{FOXH}^{-/-}$cells (pink) can contribute extensively to all tissues except gut tissues. The most anterior foregut region contains a few FoxH1 $1^{-/-}$cells, whereas the remaining gut is exclusively composed of wild-type (blue) cells. Interestingly, in this embryo a fragmentary notochord (nc) composed of $\mathrm{FOxH} 1^{-/-}$ES cells was observed in the anterior region, but this notochord does not extend to posterior regions. The significance of this is unclear. Abbreviations are visceral endoderm (ve) and definitive endoderm (de).

cells to the gut suggests that FoxH1 is required for formation of the definitive endoderm. Similarly, we evaluated gut epithelium in chimeric embryos derived from the introduction of FoxH1 $1^{-/-}$ES cells into a wild-type background. We found that the foregut, midgut, and hindgut were made up almost exclusively of wild-type cells (Fig. 8G-J), although a few FoxH1 ${ }^{-/-}$cells contributed to the most anterior region of the foregut (Fig. 8J). In contrast $\mathrm{FOXH1}^{+/+}$ES cells contributed efficiently to all regions of the gut (Fig. $8 \mathrm{C}-\mathrm{E}$ ). These results suggested that FoxH1 is important for development of the mouse definitive endoderm. To confirm this, we examined the expression pattern of the homeobox gene Hex, which 
marks the earliest definitive endoderm to emerge from the APS (Thomas et al. 1998; Martinez Barbera et al. 2000). At E8.5, Hex was expressed in the ventral foregut endoderm of wild-type embryos (Fig. 9A). In contrast, in FoxH1 mutant embryos, no Hex was detected in the involuting gut region, whereas Hex expression in the allantois (Thomas et al. 1998), a tissue not derived from the APS, was unaffected by loss of FoxH1. Altogether, these data demonstrate that FoxH1 is required for formation of the definitive endoderm.

\section{Discussion}

FoxH1 mutant embryos lack APS

During gastrulation in the mouse, patterning of the APS gives rise to the prospective node and definitive endoderm, both of which play a major role in establishing the body plan of the embryo. Cells arising from the node form the prechordal plate mesoderm and the notochord, which in turn emit signals required for D-V patterning of the neural tube and potentially the gut (Davidson and Tam 2000). In addition, the node may elaborate a signal that contributes to establishment of the $\mathrm{L}-\mathrm{R}$ axis. The APS also gives rise to the definitive endoderm (Lawson et al. 1991; Wells and Melton 1999). The molecular pathways that control formation of the APS are ill-defined. Here, we show that mice deficient in FoxH1, a transcriptional partner for Smad proteins, fail to form notochord, prechordal plate mesoderm, and definitive endoderm, reflecting a failure to establish the APS.

Foxa2 encodes a forkhead transcription factor that is required during mouse development for formation of the node, midline structures, and definitive endoderm (Ang and Rossant 1994; Weinstein et al. 1994). Prior to gastrulation, Foxa2 expression is restricted to the visceral
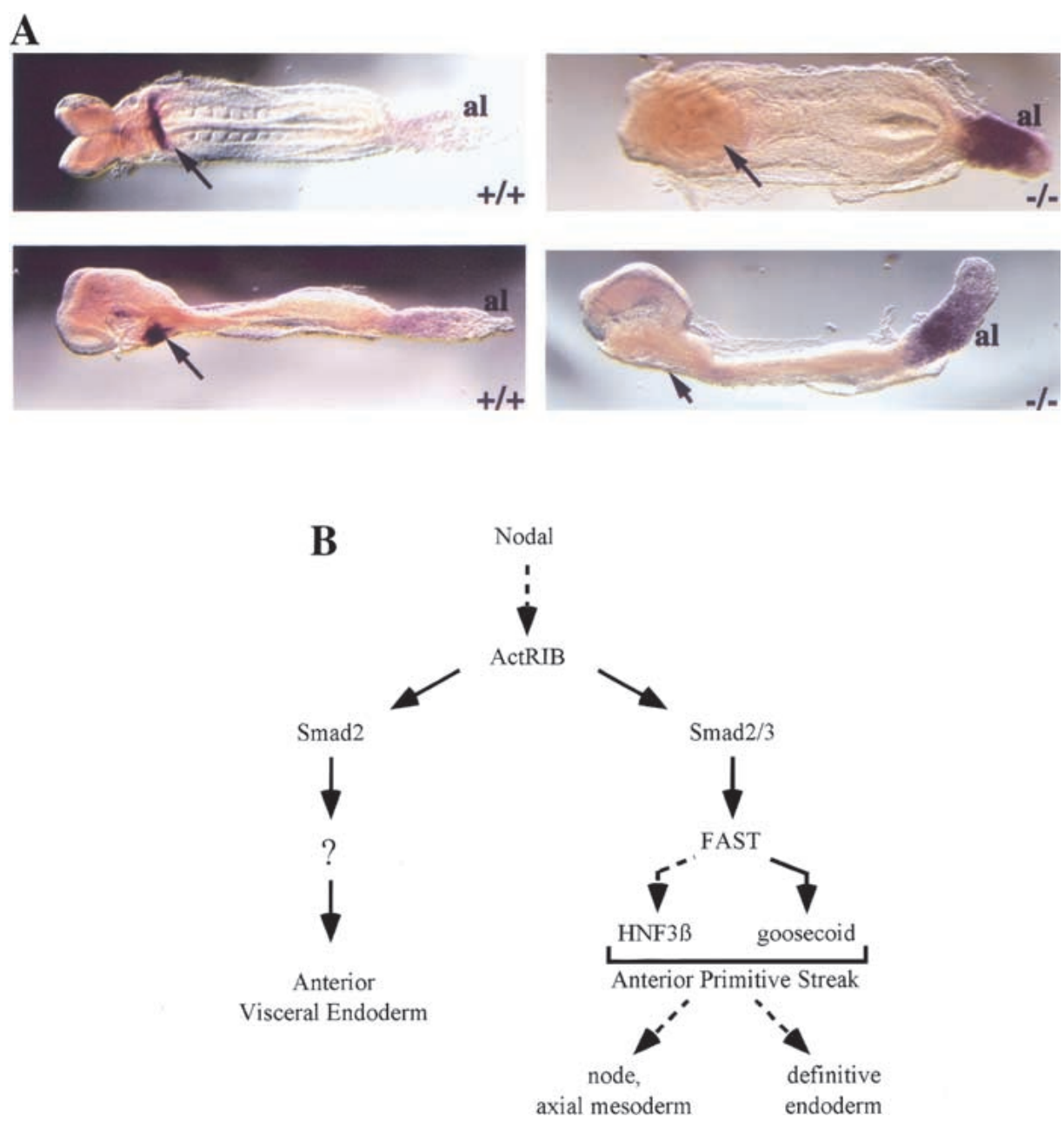

Figure 9. FoxH1 is required for formation of definitive endoderm. (A) Hex expression in wild-type $(+/+)$ and FoxH1 mutant $(-/-)$ embryos at E8.5 is shown in ventral (top panels) and lateral (bottom panels) views. Hex expression, which marks the definitive endoderm in the foregut invagination (arrow), is absent in FoxH1 mutants, whereas expression in the allantois (al) is present in both embryos. (B) A model for FoxH1 function during gastrulation in the mouse. Nodal-regulated signaling pathways that function during gastrulation in the mouse are shown. Dotted lines indicate genetic interactions, whereas solid lines show direct molecular interactions. 
endoderm and is not expressed in the epiblast. This contrasts with the widespread expression of FoxH1 (Filosa et al. 1997). During gastrulation Foxa2 expression is initiated in the epiblast at the anterior end of the primitive streak (Ang et al. 1993; Monaghan et al. 1993; Ruiz i Altaba et al. 1993), and by late streak stages it is expressed in the node, prospective floor plate of the neural tube, and the axial mesendoderm. However, in the absence of FoxH1, we observed no expression of Foxa2. Furthermore, the FoxH1 type I mutant phenotype is remarkably similar to the Foxa2 mutant embryonic phenotype. Together, these results suggest that FoxH1 functions upstream of Foxa2 in a genetic pathway that is required for formation of the APS and its derivatives (Fig. 9B).

Previous studies in Xenopus using FoxH1-repressor and -activator fusion proteins (FoxH1-En ${ }^{\mathrm{R}}$ and FoxH1VP16) have suggested that FoxH1 is required for general mesoderm specification (Watanabe and Whitman 1999). Our mutants, however, clearly exhibit normal formation of paraxial, lateral, and extraembryonic mesoderm. Recently, the zebrafish mutant schmalspur (sur), which exhibits cyclopia and an absence or reduction of the floorplate and prechordal plate, was found to encode point mutations of the zebrafish homolog of FoxH1 (Pogoda et al. 2000; Sirotkin et al. 2000). In contrast to the mouse, the phenotype of sur mutants is highly variable, and homozygous mutant adults can be viable and fertile. How zebrafish FoxH1 functions to control axial mesoderm formation is unclear; however, these results indicate that FoxH1 may play a conserved role in patterning the organizer in fish and mice.

\section{FoxH1 is required for formation}

\section{of the definitive endoderm}

The definitive or embryonic endoderm primarily originates from cells in the APS, which migrate anteriorly to displace visceral endoderm cells and form the embryonic gut (Lawson et al. 1991; Wells and Melton 1999). However, in the absence of definitive endoderm, gut invaginations can initiate, but are populated by the visceral endoderm (Dufort et al. 1998). Analysis of chimeric embryos revealed that $\mathrm{FOXH}^{-/-}$cells are unable to contribute to the definitive endoderm, regardless of whether the FoxH1-/- cells are provided by a morula or by ES cells, and in FoxH1 mutant embryos we observed no definitive endoderm formation. Thus, FoxH1 is required for specification of definitive endoderm, and the foregut and hindgut invaginations in the FoxH1 null embryos are likely populated by the visceral endoderm. Since definitive endoderm contributes to patterning of the forebrain (Martinez-Barbera et al. 2000), the defective anterior development observed in the FoxH1 mutants could be caused by loss of definitive endoderm as well as the prechordal plate and notochord. Recently, chimeric analysis showed that Smad2-deficient ES cells contribute poorly to the definitive endoderm, similar to FoxH1-deficient cells (Tremblay et al. 2000). These results are consistent with molecular studies showing that Smad2 mediates transcriptional activation of FoxH1 target elements and suggest that FoxH1-dependent specification of endoderm is dependent on Smad2 function. Interestingly, chimeric analysis has shown that Foxa2 is also required for formation of the foregut and midgut, consistent with Foxa2 functioning downstream of FoxH1 in the anterior streak. However, in contrast to FoxH1, Foxa2 is not required for hindgut development. Since regions in the primitive streak and lateral to the node can give rise to definitive endoderm that may contribute to the hindgut (Lawson et al. 1991; Dufort et al. 1998), these data suggest that FoxH1 may function in a broader domain than Foxa2 to specify the entire definitive endoderm. Whether FoxH1 might also regulate subsequent A-P patterning of the endoderm is unclear. Recently FGF, which is secreted from the primitive streak mesoderm, was shown to pattern definitive endoderm and induce organ-specific gene expression (Wells and Melton 2000). Of note, both TGF $\beta$ and activin had little patterning activity, suggesting that early A-P patterning of the endoderm may not involve FoxH1.

\section{FoxH1 is not required for specification of the AVE}

The anterior visceral endoderm (AVE) defines a domain within the visceral endoderm that is postulated to provide signals that initiate A-P patterning and head formation (Beddington and Robertson 1999). The AVE arises from a group of cells at the distal tip of the egg cylinder that moves toward the anterior side of the embryo to establish the earliest known demarcation of the embryonic axes. The AVE is proposed to establish an A-P axis by suppressing mesoderm formation and inducing anterior neuroectoderm in the epiblast. Nodal, ActRIB, and Smad2 have been implicated in formation of the AVE. Chimeric studies in which wild-type ES cells were used to rescue nodal-deficient embryos demonstrated that nodal is required in the visceral endoderm for development of anterior neural structures (Varlet et al. 1997). Similar analysis of ActRIB-deficient embryos suggests that this activin type I receptor is also required to pattern the visceral endoderm (Gu et al. 1998). In addition, two Smad2 mutant alleles, Smad2 Robm1 and Smad2 ${ }^{\text {Rex2 }}$, cause complete loss of the AVE and A-P patterning, and consequently the entire epiblast differentiates into extraembryonic mesoderm (Waldrip et al. 1998; Heyer et al. 1999). In contrast, in FoxH1 null embryos, the AVE markers Hex and Cer-1 are expressed appropriately, and mesoderm formation initiates at the posterior side in both type I and type II mutant embryos. Furthermore, at later stages in type I mutants the midbrain/hindbrain marker En-2 is expressed, and the forebrain marker Six-3 is also expressed, albeit at reduced levels. Thus, despite defects in anterior development, initial A-P patterning remains intact. Finally, chimeric embryos, in which the visceral endoderm is composed of $\mathrm{FOXH1}^{-/-}$cells, develop normal anterior structures. These results indicate that FoxH1 is not required for AVE formation.

FoxH1 is expressed weakly, however, throughout the visceral endoderm, and $25 \%$ of FoxH1 mutant embryos 
displayed a constriction at the extraembryonic/embryonic junction. What causes these constrictions is unclear, but they may arise as a result of reduced proliferation of the visceral endoderm during expansion of the embryonic epiblast. Importantly, we observed that both brachyury and nodal were expressed on the presumptive posterior side of severely pinched type II embryos, indicating that initiation of A-P patterning was preserved. Interestingly, Hamada and colleagues have also analyzed deletion of the FoxH1 gene (Yamamoto et al. 2001), but report a spectrum of visceral endoderm (VE) defects that are more severe than we observed. These defects can lead to disruption of A-P axis formation and appear to reflect a failure to rotate the AVE to the anterior region of the $\mathrm{VE}$, rather than a loss of AVE per se. It is unclear why there are differences in the severity of the VE defects between our studies. However, it is possible that rotation of the AVE and establishment of the A-P axis is linked to proliferative expansion of the VE and that this latter pathway is differentially affected in different genetic backgrounds by loss of FoxH1. Analysis of the relationship between constrictions at the extraembryonic/ embryonic junction and rotation of the AVE requires further investigation. Nevertheless, our studies indicate that FoxH1 is not critical for specifying the AVE, and we propose that other Smad partners probably play a critical role in patterning these cells (Fig. 9B). Thus, distinct nuclear Smad DNA-binding partners likely function in different cell types to mediate unique transcriptional responses to nodal and nodal-related signals.

\section{A signaling pathway required for induction of the node}

The signaling pathway that regulates FoxH1 function during axial mesoderm formation remains unclear. Nodal is the primary candidate because it is expressed in the posterior epiblast at the onset of gastrulation and its expression becomes restricted to the node during gastrulation. Although nodal mutants arrest prior to gastrulation (Zhou et al. 1993; Conlon et al. 1994), introduction of wild-type ES cells into nodal mutant blastocysts is able to partially rescue gastrulation defects. Some of these chimeric embryos display axial defects with fused somites, and nodal ${ }^{-/-}$cells are impaired in their ability to contribute to midline structures, indicating that nodal is required for node morphogenesis (Varlet et al. 1997). Furthermore, trans-heterozygote embryos of Smad2 and nodal display severe craniofacial defects, which include cyclopia, suggesting that Smad2 and nodal play a role in patterning the midline of the embryo, at least in anterior regions (Nomura and Li 1998). Together, these results indicate that nodal induces midline structures via a Smad-mediated pathway that interacts with FoxH1.

The direct gene targets of FoxH1 that function to regulate formation of the node are only beginning to be unraveled. It is unclear whether Foxa2 is a direct target of the FoxH1 transcription factor in the epiblast. Our analysis of the Foxa2 gene sequence revealed no consensus
FoxH1-binding motifs, and analysis of reporter gene assays using fragments of the Foxa2 promoter failed to reveal the presence of any TGF $\beta$ or FoxH1-dependent response elements (data unpubl.). Similarly, an activin-responsive element in the Xenopus Foxa2 gene does not contain consensus FoxH1-binding sites (Howell and Hill 1997). In contrast, previous analysis of the goosecoid promoter showed that FoxH1 directly regulates a TGF $/$ activin response element found in both the Xenopus and mouse genes (Labbé et al. 1998). The absence of goosecoid expression in FoxH1-deficient embryos is consistent with the notion that goosecoid is a direct target for FoxH1-dependent transcription during anterior patterning of the streak. Importantly, goosecoid expression in the APS is not dependent on Foxa2 (Dufort et al. 1998). Furthermore, although goosecoid on its own is not essential for node formation, loss of goosecoid enhances midline defects in Foxa2 ${ }^{+/-}$embryos (Filosa et al. 1997). We therefore propose a model (see Fig. 9B) in which goosecoid may cooperate with Foxa2 as part of a transcriptional program that is coordinately regulated by FoxH1 to specify the APS and its derivatives, the node, axial mesoderm, and definitive endoderm.

The goosecoid promoter may also be regulated by the homeodomain proteins Mixer and Milk, which can function as Smad partners (Germain et al. 2000). Although homologs have not been identified in the mouse, in the frog these genes are expressed in mesendodermal cells, which in the mouse are derivatives of the node (Ecochard et al. 1998; Henry and Melton 1998). We therfore propose that FoxH1 initiates goosecoid expression in the anterior streak and node and that other Smad partners, such as Mixer and Milk, may cooperate at later stages to maintain expression in mesendodermal derivatives. Lefty and nodal also contain FoxH1-binding sites that can be transcriptionally activated in vitro by activin-like signals. Whether in the mouse FoxH1 functions to initiate or maintain the activity of these genes, as suggested by studies in zebrafish (Pogoda et al. 2000; Sirotkin et al. 2000), is currently under investigation. Accordingly, FoxH1 may mediate a unique gene expression program in response to TGF $\beta$ family signaling that specifies formation of the node. The temporal restriction of FoxH1 expression to the early embryo may ensure that this program is not activated promiscuously during later development.

The signals within the APS and node that dictate the decision between axial mesoderm and endoderm are unclear. Recent studies have demonstrated that Smad2 ${ }^{-/}$ cells are incapable of forming definitive endoderm, but contribute to axial mesoderm (Tremblay et al. 2000). This contrasts with the requirement for FoxH1 in both pathways. Because nodal activates Smad2 and Smad3 (Kumar et al. 2000), which both bind FoxH1, these results suggest that $\mathrm{Smad} 3$ may substitute for Smad2 as a FoxH1 partner during specification of axial mesoderm, but is unable to replace Smad2 function during formation of the definitive endoderm. Interestingly, molecular analysis of Smad-dependent regulation of FoxH1 target genes has shown that some of these can be activated by 
either Smad2 or Smad3. In contrast, FoxH1-dependent elements in the gsc promoter are positively regulated only by Smad2, whereas Smad3 blocks ligand-dependent induction (Labbé et al. 1998; Nagarajan et al. 1999). The basis for this functional difference likely resides in the ability of Smad3 to interact with DNA. It is therefore intriguing to speculate that the balance between Smad2 and Smad3 activation in cells of the APS may regulate cell fate determination, by controlling specific FoxH1dependent transcriptional responses.

\section{Materials and methods}

\section{Generation of FoxH1 mutant mice}

A FoxH1 mouse cDNA clone (Labbé et al. 1998) was used to screen a $129 \mathrm{~Sv} / \mathrm{J}$ mouse genomic $\lambda$ phage library. Four genomic clones were isolated, spanning $\sim 28 \mathrm{~kb}$ of sequence. To construct a positive/negative targeting vector, a $3.6-\mathrm{kb}$ SalI/EcoRI fragment from the $5^{\prime}$ end of the gene and a $5.5-\mathrm{kb} E c o \mathrm{RI} / \mathrm{BamH} 1$ fragment from the $3^{\prime}$ end of the gene were cloned into the vector pPNTloxPneo. The resulting vector was linearized with NotI and electroporated into R1 ES cells as described (Hogan et al. 1994). Drug-resistant colonies were picked into 96-well plates and screened by Southern blotting as described (Hogan et al. 1994). Briefly, DNA was digested with HindIII/NotI or BamH1, electophoresed overnight at $30 \mathrm{~V}$ on an $0.8 \%$ agarose gel, transferred to Zetaprobe GT (Bio-Rad), and probed according to the manufacturer's protocol. BamH1-digested DNA and HindIII/ NotI-digested DNA were probed with probe \#1 and \#2, respectively (see Fig. 1). Approximately 1 in 14 drug-resistant colonies contained correctly targeted alleles. Correctly targeted clones were aggregated with CD-1 morula to generate germline chimera as described (Hogan et al. 1994). $F_{1}$ progeny were genotyped by Southern blot as described above.

\section{Genotyping procedures}

Offspring and embryos were maintained on a 129/CD-1 background and were genotyped using a PCR-based strategy. For E6.5 and E7.5 embryos, the ectoplacental cone (EPC) was removed and cultured in gelatin-coated 96-well plates for 4-7 d in DMEM plus $10 \%$ fetal bovine serum (GIBCO) and antibiotics (penicillin and streptomycin). The EPC colonies were washed with PBS and lysed overnight at $55^{\circ} \mathrm{C}$ in $40 \mu \mathrm{L}$ of buffer $(50 \mathrm{mM} \mathrm{KCl}, 10$ $\mathrm{mM}$ Tris- $\mathrm{HCl}$ at $\mathrm{pH} 8.8,2.5 \mathrm{mM} \mathrm{MgCl}_{2}, 0.1 \mathrm{mg} / \mathrm{mL}$ gelatin, $0.45 \%$ NP- $40,0.45 \%$ Tween 20 ) containing proteinase $\mathrm{K}$ at 1 $\mathrm{mg} / \mathrm{mL}$. For older embryos the visceral yolk sac was removed, and for chimeric embryos the parietal endoderm was isolated and lysed as described above. Prior to PCR, samples were heatinactivated for $10 \mathrm{~min}$ at $95^{\circ} \mathrm{C}$. A $1.5-\mu \mathrm{L}$ aliquot was used for PCR amplification containing $0.2 \mathrm{mM}$ dNTPs, $0.5 \mu \mathrm{M}$ of each primer, 0.6 U HotStar Taq (Qiagen), and the supplied buffer. Following initial denaturation and enzyme activation at $95^{\circ} \mathrm{C}$ for $15 \mathrm{~min}$, amplification was conducted for 40 cycles at $95^{\circ} \mathrm{C}$ for $30 \mathrm{sec}, 60^{\circ} \mathrm{C}$ for $30 \mathrm{sec}$, and $72^{\circ} \mathrm{C}$ for $30 \mathrm{sec}$. Two different sets of primers were used throughout this study. The first set used CreR (5'-TGGCTGGACGTAAACTCCTC-3'), R4 (5'AAACCCACCATCTCTCACCAG-3'), and F5 (5'-AACCGGT GGTACCTGTGATAC-3') to amplify 219-bp and 379-bp fragments from the mutant and wild-type alleles, respectively. The second set used CreR, R6 (5'-AGTCAGGTCAGGGATGC GTG- $\left.3^{\prime}\right)$, and F6 (5'-GCGTGAGCTGTGCTGGTTCA-3') to amplify 300-bp and 384-bp fragments from the mutant and wild- type alleles, respectively. The products were analyzed on a $1.8 \%$ agarose gel.

\section{In situ hybridization and histology}

For histology, embryos were fixed in $3.7 \%$ formaldehyde, dehydrated through an ethanol series, and embedded in paraffin. Paraffin blocks were sectioned at $6 \mu \mathrm{m}$, mounted onto glass slides, dewaxed, and stained with eosin and hematoxylin. For whole mount in situ hybridizations, embryos were fixed overnight at $4^{\circ} \mathrm{C}$ in $4 \%$ paraformaldehyde in PBS, dehydrated in methanol, and stored at $-20^{\circ} \mathrm{C}$. For hybridizations, embryos were processed as described at www.hhmi.ucla.edu/derobertis/ (Belo et al. 1997). Briefly, genotyped embryos were rehydrated in PBSw (PBS plus $0.1 \%$ Tween 20$)$ and incubated in proteinase $\mathrm{K}(4.5$ $\mu \mathrm{g} / \mathrm{mL}$ in PBSw) for $3 \mathrm{~min}$ (E6.5), $5 \mathrm{~min}$ (E7.5), or $6 \mathrm{~min}$ (E8.5) at room temperature. Digestion was stopped with $2 \mathrm{mg} / \mathrm{mL}$ glycine in PBSw, and the embryos were refixed in 4\% paraformaldehyde with $0.2 \%$ glutaraldehyde in PBSw. Embryos were washed and hybridized overnight at $70^{\circ} \mathrm{C}$ with approximately $200 \mathrm{ng} / \mathrm{mL}$ of digoxigenin-labeled riboprobe in hybridization solution ( $50 \%$ formamide, $1 \%$ blocking agent [Boehringer], $5 \times$ SSC at $\mathrm{pH} 7.5,1 \mathrm{mg} / \mathrm{mL}$ yeast tRNA, $0.1 \mathrm{mg} / \mathrm{mL}$ heparin, $0.1 \%$ Tween 20, 0.1\% CHAPS, and 5 mM EDTA). Embryos were washed for $5 \mathrm{~min}$ in fresh hybridization solution, twice for 30 min in $2 \times$ SSC plus $0.1 \%$ CHAPS at $70^{\circ} \mathrm{C}$, for $30 \mathrm{~min}$ in $\mathrm{MAB}$ at room temperature, twice for $30 \mathrm{~min}$ in $\mathrm{MAB}$ at $70^{\circ} \mathrm{C}$, and twice for $10 \mathrm{~min}$ in PBSw. Embryos were then incubated overnight at $4^{\circ} \mathrm{C}$ with anti-dig alkaline phosphatase antibody $(1: 10,000$ dilution in PBSw containing $1 \%$ Boehringer blocking agent and $10 \%$ heat-inactivated goat serum). Embryos were washed 5 times for $45 \mathrm{~min}$ in PBSw with $0.1 \% \mathrm{BSA}$ and twice for $10 \mathrm{~min}$ in AP1 buffer (0.1 M NaCl, 0.1 M Tris- $\mathrm{HCl}$ at $\mathrm{pH} 9.5$, and 50 $\mathrm{mM} \mathrm{MgCl} 2$ ). Embryos were stained in BM Purple (Boehringer) at room temperature.

\section{ES cell derivation; generation and analysis} of chimeric embryos

KT4 cells were described previously (Tremblay et al. 2000). FoxH1 mutant ES cell lines were isolated from blastocysts from FoxH1 heterozygote matings as described. Briefly, blastocysts were flushed and cultured overnight, transferred to a 96-well plate (1 blastocyst/well) containing mitomycin-treated mouse embryonic fibroblasts, and cultured 4-5 d in DMEM containing $15 \%$ fetal bovine serum, $0.1 \mathrm{mM}$ nonessential amino acids, 1 $\mathrm{mM}$ sodium pyruvate, $0.1 \mathrm{mM} \beta$-mercaptoethanol, $2 \mathrm{mM} \mathrm{L}$ glutamine, penicillin, streptomycin, and LIF. The ICM outgrowths were trypsinized and cultured until ES cell colonies developed. Colonies with good morphology were genotyped as described above.

Chimeric embryos were generated by aggregation with morulae as described (Hogan et al. 1994). Morulae were obtained from FoxH1 heterozygote matings or from ICR/Tg(Rosa26)RSor (Jackson Laboratories) matings. Aggregates were implanted into recipient females, and the chimeric embryos were dissected at E8.5-E9.5. Embryos were stained for $\beta$-gal expression and then fixed in formalin and paraffin-sectioned as described above. Sections were dewaxed and counterstained with Nuclear Fast Red.

\section{Acknowledgments}

We thank M. Crackower, P. Hunter, the Transgenic Mouse Facilities at the Hospital for Sick Children, Mount Sinai Hospital, and K. Harpal for advice and excellent technical assistance dur- 
ing this work. We also thank R. Beddington for the hex in situ probe and E. DeRobertis for the Gsc and Cer-1 in situ probes. In addition, we thank E.J. Robertson for her insightful comments and Hiroshi Hamada for communicating results prior to publication. This work was supported by grants to J.L.W. from the Canadian Institutes of Health Research (CIHR) and the National Cancer Institute of Canada, with funds from the Terry Fox run. P.A.H. was supported by an MRC Centennial Fellowship and a Canadian Association of Gastroenterology Fellowship. L.A. is a CIHR Scholar, J.R. is a CIHR Distinguished Investigator, and J.L.W. is a CIHR Investigator.

The publication costs of this article were defrayed in part by payment of page charges. This article must therefore be hereby marked "advertisement" in accordance with 18 USC section 1734 solely to indicate this fact.

\section{References}

Ang, S.-L. and Rossant, J. 1994. HNF-3 $\beta$ is essential for node and notochord formation in mouse development. Cell 78: 561574.

Ang, S.-L., Wierda, A., Wong, D., Stevens, K.A., Cascio, S., Rossant, J., and Zaret, K.S. 1993. The formation and maintenance of the definitive endoderm lineage in the mouse: Involvement of HNF3/forkhead proteins. Development 119: $1301-1315$.

Ang, S.-L., Jin, O., Rhinn, M., Daigle, N., Stevenson, L., and Rossant, J. 1996. A targeted mouse Otx2 mutation leads to severe defects in gastrulation and formation of axial mesoderm and to deletion of rostral brain. Development 122: 243-252.

Attisano, L. and Wrana, J.L. 2000. Smads as transcriptional comodulators. Curr. Opin. Cell Biol. 12: 235-243.

Bachiller, D., Klingensmith, J., Kemp, C., Belo, J.A., Anderson, R.M., May, S.R., McMahon, J.A., McMahon, A.P., Harland, R.M., Rossant, J., and DeRobertis, E.M. 2000. The organizer factors chordin and noggin are required for mouse and forebrain development. Nature 403: 658-661.

Beddington, R.S.P. and Robertson, E.J. 1989. An assessment of the developmental potential of embryonic stem cells in the midgestation mouse embryo. Development 105: 733-737.

- 1999. Axis development and early asymmetry in mammals.Cell 96: 195-209.

Belo, J.A., Bouwmeester, T., Leyns, L., Kertesz, N., Gallo, M., Folliettie, M., and DeRobertis, E.M. 1997. Cerberus-like is a secreted factor with neuralizing activity expressed in the anterior primitive endoderm of the mouse gastrula. Mech. Dev. 68: 45-57.

Chen, X., Rubock, M.J., and Whitman, M. 1996. A transcriptional partner for MAD proteins in TGF- $\beta$ signaling. Nature 383: 691-696.

Conlon, F.L., Lyons, K.M., Takaesu, N., Barth, K.S., Kispert, A., Herrmann, B., and Robertson, E.J. 1994. A primary requirement for nodal in the formation and maintenance of the primitive streak in the mouse. Development 120: 19191928.

Davidson, B.P. and Tam, P.P.L. 2000. The node of the mouse embryo. Curr. Biol. 10: R617-R619.

Dufort, D., Schwartz, L., Harpal, K., and Rossant, J. 1998. The transcription factor $H N F 3 \beta$ is required in visceral endoderm for normal primitive streak morphogenesis. Development 125: 3015-3025.

Ecochard, V., Cayrol, C., Rey, S., Foulquier, F., Caillol, D., Lemaire, P., and Duprat, A.M. 1998. A novel Xenopus mix-like gene milk involved in the control of the endomesodermal fates. Development 125: 2577-2585.

Filosa, S., Rivera-Pérez, J.A., Gómez, A.P., Gansmuller, A., Sasaki, H., Behringer, R.R., and Ang, S.-L. 1997. goosecoid and $H N F-3 \beta$ genetically interact to regulate neural tube patterning during mouse embryogenesis. Development 124: 2843-2854.

Germain, S., Howell, M., Esslemont, G.M., and Hill, C.S. 2000. Homeodomain and winged-helix transcription factors recruit activated Smads to distinct promoter elements via a common Smad interaction motif. Genes \& Dev. 14: 435451.

Gu, Z., Nomura, M., Simpson, B.B., Lei, H., Feijen, A., van den Eijnden-van Raaij, J., Donahoe, P.K., and Li, E. 1998. The type I activin receptor ActRIB is required for egg cylinder organization and gastrulation in the mouse. Genes \& Dev. 12: $844-857$.

Harland, R. and Gerhart, J. 1997. Formation and function of Spemann's organizer. Annu. Rev. Cell Dev. Biol. 13: 611667.

Harvey, R.P. 1998. Links in the left/right axial pathway. Cell 94: 273-276.

Henry, G.L. and Melton, D.A. 1998. Mixer, a homeobox gene required for endoderm development. Science 281: 91-96.

Hermesz, E., Mackem, S., and Mahon, K.A. 1996. Rpx: A novel anterior-restricted homeobox gene progressively activated in the prechordal plate, anterior neural plate and Rathke's pouch of the mouse embryo. Development 122: 41-52.

Heyer, J., Escalante-Alcalde, D., Lia, M., Boettinger, E., Edelmann, W., Stewart, C.L., and Kucherlapati, R. 1999. Postgastrulation Smad2-deficient embryos show defects in embryo turning and anterior morphogenesis. Proc. Natl. Acad. Sci. USA 96: 12595-12600.

Hogan, B.L.H., Beddington, R.S.P., Constantini, F., and Lacy, E. 1994. Manipulating the mouse embryo: A laboratory manual. Cold Spring Harbor Laboratory Press, Cold Spring Harbor, NY.

Hoodless, P.A., Tsukazaki, T., Nishimatsu, S.-I., Attisano, L., Wrana, J.L., and Thomsen, G.H. 1999. Dominant-negative Smad2 mutants inhibit activin/Vg1 signaling and disrupt axis formation in Xenopus. Dev. Biol. 207: 364-379.

Howell, M. and Hill, C. 1997. XSmad2 directly activates the activin-inducible dorsal mesoderm gene XFKH1 in Xenopus embryos. EMBO J. 16: 7411-7421.

Klüppel, M., Hoodless, P.A., Wrana, J.L., and Attisano, L. 2000. Mechanisms and biology of signaling by serine/threonine kinase receptors for the TGF $\beta$ superfamily. In Protein kinase functions (ed. J. Woodgett), pp. 303-356. Oxford University Press, Oxford, UK.

Kumar, A., Novoselov, V., Celeste, A.J., Wolfman, N.M., ten Dijke, P., and Kuehn, M.R. 2001. Nodal signaling uses activin and Transforming Growth Factor- $\beta$ receptor-regulated Smads. J. Biol. Chem. 276: 656-661.

Labbé, E., Silvestri, C., Hoodless, P.A., Wrana, J.L., and Attisano, L. 1998. Smad2 and Smad3 positively and negatively regulate TGF $\beta$-dependent transcription through the forkhead DNA binding protein, FAST2. Mol. Cell 2: 109-120.

Lawson, K.A., Meneses, J.J., and Pedersen, R.A. 1991. Clonal analysis of epiblast fate during germ layer formation in the mouse embryo. Development 113: 891-911

Liu, B., Dou, C.-L., Prabhu, L., and Lai, E. 1999. FAST-2 is a mammalian winged-helix protein which mediates transforming growth factor $\beta$ signals. Mol. Cell. Biol. 19: 424-430.

Martinez Barbera, J.P., Clements, M., Thomas, P., Rodriguez, T., Meloy, D., Kioussis, D., and Beddington, R.S. 2000. The homeobox gene Hex is required in definitive endodermal 
tissues for normal forebrain, liver and thyroid formation. Development 127: 2433-2445.

Monaghan, A.P., Kaestner, K.H., Grau, E., and Schütz, G. 1993. Postimplantation expression patterns indicated a role for the mouse forkhead/HNF- $3 \alpha, \beta$ and $\gamma$ genes in determination of the definitive endoderm chordamesoderm and neuroectoderm. Development 119: 567-578.

Nagarajan, R.P., Liu, J., and Chen, Y. 1999. Smad3 inhibits transforming growth factor- $\beta$ and activin signaling by competing with Smad4 for FAST-2 binding. I. Biol. Chem. 274: 31229-31235.

Nomura, M. and Li, E. 1998. Smad2 role in mesoderm formation, left-right patterning and craniofacial development. $\mathrm{Na}$ ture 393: 786-790.

Osada, S.-I., Saijoh, Y., Frisch, A., Yeo, C.-Y., Adachi, H., Watanabe, M., Whitman, M., Hamada, H., and Wright, C.V.E. 2000. Activin/nodal responsiveness and symmetric expression in a Xenopus nodal-related gene converge on a FASTregulated module in intron 1. Development 127: 2503-2514.

Pogoda, H.-M., Solnica-Krezel, L., Driever, W., and Meyer, D. 2000. The zebrafish forkhead transcription factor FoxH1/ Fast 1 is a modulator of nodal signaling required for organizer function. Curr. Biol. 10: 1041-1049.

Ruiz i Altaba, A., Prezioso, V.R., Darnell, J.E., and Jessell, T.M. 1993. Sequential expression of HNF-3 $\beta$ and HNF-3 $\alpha$ by embryonic organizing centers: The dorsal lip/node, notochord and floorplate. Mech. Dev. 44: 91-108.

Saijoh, Y., Adachi, H., Sakuma, R., Yeo, C.-Y., Yashiro, K., Watanabe, M., Hashiguchi, H., Mochida, K., Ohishi, S., Kawabata, M., et al. 2000. Left-right asymmetric expression of lefty2 and nodal is induced by a signaling pathway that includes the transcription factor FAST2. Mol. Cell 5: 35-47.

Schier, A.F. and Shen, M.M. 1999. Nodal signaling in vertebrate development. Nature 403: 385-389.

Shawlot, W. and Behringer, R.R. 1995. Requirement for Lim1 in head-organizer function. Nature 374: 425-430.

Shiratori, H., Sakuma, R., Watanabe, M., Hashiguchi, H., Mochida, K., Sakai, Y., Nishino, J., Saijoh, Y., Whitman, M., and Hamada, H. 2001. Two-step regulation of left-right asymmetric expression of Pitx2: Initiation by nodal signaling and maintenance by Nkx2. Mol. Cell 7: 137-149.

Sirotkin, H.I., Gates, M.A., Kelly, P.D., Schier, A.F., and Talbot, W.S. 2000. fast1 is required for the development of dorsal axial structures in zebrafish. Curr. Biol. 10: 1051-1054.

Thomas, P.Q., Brown, A., and Beddington, R.S.P. 1998. Hex: A homeobox gene revealing peri-implantation asymmetry in the mouse embryo and an early transient marker of endothelial cell precursors. Development 125: 85-94.

Tremblay, K.D., Hoodless, P.A., Bikoff, E.K., and Robertson, E.J. 2000. Formation of the definitive endoderm in mouse is a Smad2-dependent process. Development 127: 3079-3090.

Varlet, I., Collingnon, J., and Robertson, E.J. 1997. nodal expression in the primitive endoderm is required for specification of the anterior axis during mouse gastrulation. Development 124: $1033-1044$

Vesque, C., Ellis, S., Lee, A., Szabo, M., Thomas, P., Beddington, R., and Placzek, M. 2000. Development of chick axial mesoderm: Specification of prechordal mesoderm by anterior endoderm-derived TGF $\beta$ family signaling. Development 127: 2795-27809.

Waldrip, W.R., Bikoff, E.K., Hoodless, P.A., Wrana, J.L., and Robertson, E.J. 1998. Smad2 signaling in extraembryonic tissues determines anterior-posterior polarity of the early mouse embryo. Cell 92: 797-808.

Watanabe, M. and Whitman, M. 1999. FAST-1 is a key maternal effector of mesoderm inducers in the early Xenopus embryo.
Development 126: 5621-5634.

Weinstein, D.C., Ruiz i Altaba, A., Chen, W.S., Hoodless, P., Prezioso, V., Jessell, T.M., and Darnell, J.E.J. 1994. The winged-helix transcription factor $H N F-3 \beta$ is required for notochord development in the mouse embryo. Cell 78: 575588 .

Weinstein, M., Yang, X., Li, C., Xu, X., Goday, J., and Deng, C.-X. 1998. Failure of egg cylinder elongation and mesoderm induction in mouse embryos lacking the tumor suppressor Smad2. Proc. Nat1. Acad. Sci. USA 95: 9378-9383.

Weisberg, E., Winnier, G.E., Chen, X., Farnsworth, C.L., Hogan, B.L.H., and Whitman, M. 1998. A mouse homologue of FAST-1 transduces TGF $\beta$ superfamily signals and is expressed during early embryogenesis. Mech. Dev. 79: 17-27.

Wells, J.M. and Melton, D.A. 1999. Vertebrate endoderm development. Annu. Rev. Cell Dev. Biol. 15: 393-410.

. 2000. Early mouse endoderm is patterned by soluble factors from adjacent germ layers. Development 127: 15631572.

Yamamoto, M., Meno, C., Sakai, Y., Shiratori, H., Mochida, K., Ikawa, Y., Saijoh, Y., and Hamada, H. 2001. The transcription factor FoxH1 (FAST) mediates Nodal signaling during anterior-posterior patterning and node formation in the mouse. Genes \& Dev. 15: 1242-1256.

Yang, Z., Roberts, E.A., and Goldstein, L.S.B. 2001. Functional analysis of mouse C-terminal kinesin motor KifC2. Mol. Cell. Biol. 21: 2463-2466.

Zhou, X., Sasaki, H., Lowe, L., Hogan, B.L.M., and Kuehn, M.R. 1993. Nodal is a novel TGF- $\beta$-like gene expressed in the mouse node during gastrulation. Nature 361: 543-547.

Zhou, S., Zawel, L., Lengauer, C., Kinzler, K.W., and Vogelstein, B. 1998. Characterization of human FAST-1, a TGF $\beta$ and activin signal transducer. Mol. Cell 2: 121-127. 


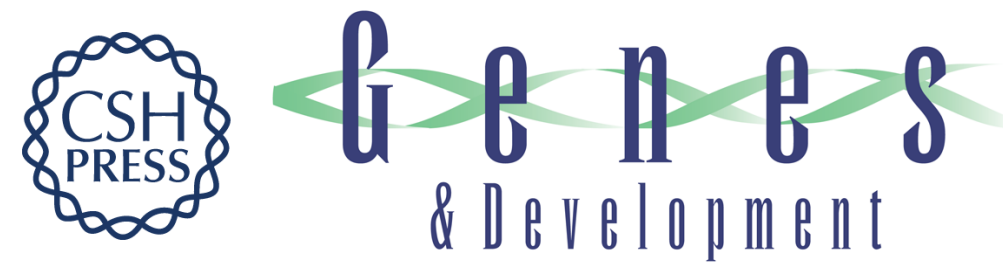

\section{FoxH1 (Fast) functions to specify the anterior primitive streak in the mouse}

Pamela A. Hoodless, Melanie Pye, Claire Chazaud, et al.

Genes Dev. 2001, 15:

Access the most recent version at doi:10.1101/gad.881501

References This article cites 53 articles, 29 of which can be accessed free at: http://genesdev.cshlp.org/content/15/10/1257.full.html\#ref-list-1

License

Email Alerting

Receive free email alerts when new articles cite this article - sign up in the box at the top Service right corner of the article or click here.

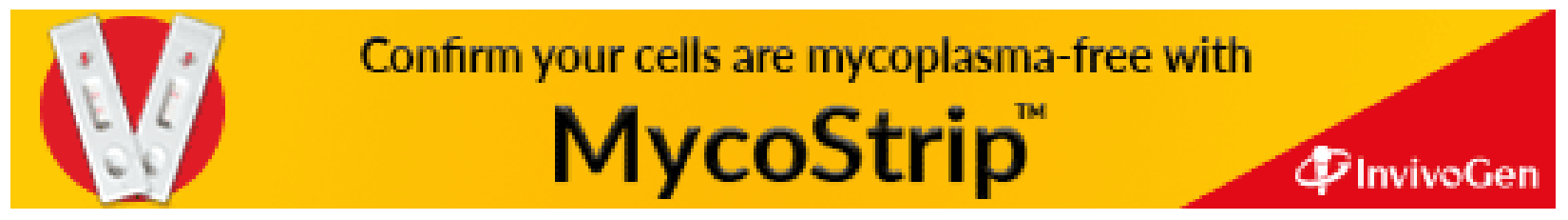

